\title{
The pre-BCR shows its negative side
}

Given the well-established role of the pre-B-cell receptor (pre-BCR) in the positive selection of $B$ cells, it comes as a surprise to learn that the pre-BCR also has a role in negative selection. A new study published in Science indicates that the pre-BCR censors the development of autoreactive $B$ cells and that the absence of this checkpoint can result in the production of antinuclear antibodies (ANAs).

The BCR repertoire is generated by recombination of the immunoglobulin heavy- and light-chain genes. Recombination starts with the heavy chain in pro-B cells, producing a pre-BCR that consists of a recombined heavy chain and an invariant surrogate light chain (SLC). Signals through the pre-BCR positively select and lead to the proliferation of pre- $\mathrm{B}$ cells that have successfully rearranged the heavy chain. Mice that are deficient for SLC have decreased numbers of immature and mature B cells owing to the absence of positive selection.
Keenan et al. observed that the serum of SLC-deficient mice contained an increased concentration of ANAs, which indicates that some $B$ cells in these mice had escaped the negative selection of BCRs that are specific for self antigens, such as DNA. Although the SLCdeficient mice had a decreased total number of splenic B cells compared with control mice, they had an increased proportion of mature CD21-CD23- B cells, and this population was shown to produce ANAs after in vitro stimulation.

It had previously been observed that ANAs frequently contain at least one basic amino acid, such as arginine, in complementaritydetermining region 3 of the heavy chain (HC-CDR3). CD21-CD23$B$ cells from SLC-deficient mice often had two or more arginines in HC-CDR3 compared with an average of one arginine in $\mathrm{CD} 21^{-} \mathrm{CD} 23^{-}$ $B$ cells from wild-type mice. In SLC-deficient CD21-CD23- B cells,

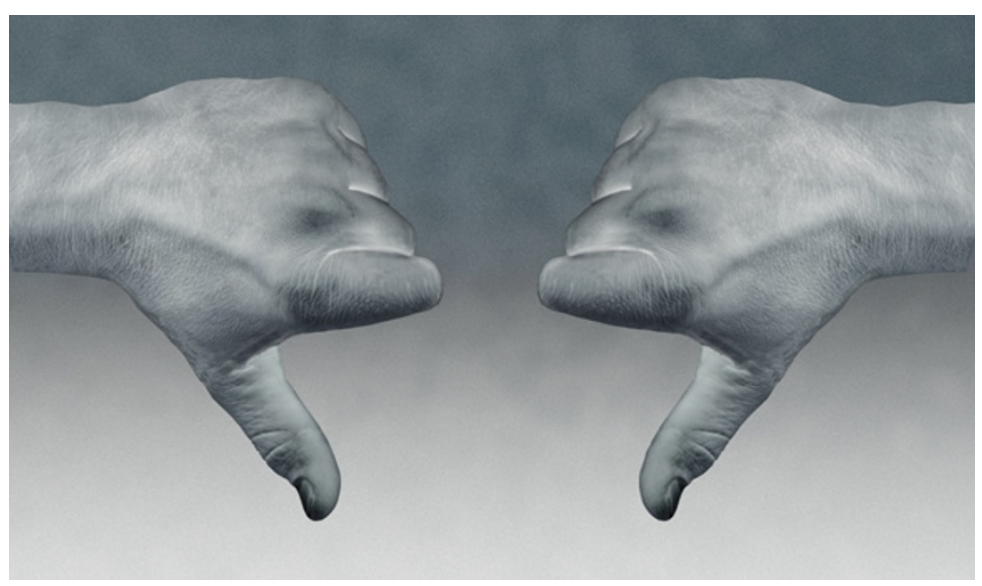

one of the arginines was preferentially encoded by the $\mathrm{D}$ (diversity) gene segment, which, based on the position of the start codon, must be translated in the infrequently used reading frame 3 (RF3) to produce arginine. The use of RF3 and RF2 was increased in SLC-deficient $\mathrm{CD} 21^{-} \mathrm{CD} 23^{-} \mathrm{B}$ cells compared with wild-type CD21-CD23- B cells, which preferentially use RF1.

By comparing HC-CDR3 sequences in pro- and pre-B cells - before and after the postulated negative-selection checkpoint mediated by the pre-BCR - the authors showed that in SLC- and light-chain-deficient B cells, the high proportion of HC-CDR3 arginines and use of RF3 in pre-B cells was similar to that of control pro-B cells (as would be expected if negative selection is absent). This contrasts with the decrease in the proportion of arginines and use of RF3 between proand pre-B cells in wild-type mice (as expected due to negative selection).

These data indicate that the pre-BCR has a role in selecting against B cells expressing a HC-CDR3 with the potential to generate ANAs. However, SLC-deficient mice have not shown any evidence of autoimmune disease, which supports the presence of multiple negative-selection checkpoints.

Kirsty Minton

ORIGINAL RESEARCH PAPER Keenan, R. A. et $a$ Censoring of autoreactive $B$ cell development by the pre-B cell receptor. Science 19 June 2008 (doi:10.1126/science.1157533) 